\title{
Improved velocity law parameterization for hot star winds (Research Note)
}

\author{
J. Krtička ${ }^{1}$ and J. Kubát ${ }^{2}$ \\ 1 Ústav teoretické fyziky a astrofyziky PřF MU, 61137 Brno, Czech Republic \\ e-mail: krticka@physics.muni.cz \\ 2 Astronomický ústav, Akademie věd České republiky, 25165 Ondřejov, Czech Republic
}

Received 9 February 2011 / Accepted 29 August 2011

\section{ABSTRACT}

\begin{abstract}
The velocity law of hot star winds is usually parameterized via the so-called beta velocity law. Although this parameterization stems from theoretical considerations, it is not the most accurate description of the wind velocity law that follows from hydrodynamical calculations. We show that the velocity profile of our hydrodynamical wind models is described much better by polynomial approximation. This approximation provides a better fit than the beta velocity law already for the same number of free parameters.
\end{abstract}

Key words. stars: winds, outflows - stars: mass-loss - stars: early-type - hydrodynamics - stars: atmospheres

Hot stars lose an important part of their mass via line-driven winds. Consequently, mass-loss rate prescriptions are an important part of evolutionary codes. Such prescriptions can be derived from theoretical modelling of hot star winds. However, any theoretical prescription should be tested against the mass-loss rates derived from observations. Although there are several possibilities for deriving wind mass-loss rates (e.g., from $\mathrm{H} \alpha$ line profiles, from ultraviolet $\mathrm{P}$ Cygni line profiles, and from radio continua), knowing of the wind velocity law is needed to derive the mass-loss rates from observations. Although there exist sophisticated hydrodynamic calculations giving the wind velocity law in a tabular form (e.g., Gräfener \& Hamann 2005; Krtička \& Kubát 2010), analytic formulae are useful in many analyses. Consequently, the question of proper velocity law parameterization is important for analysing observed hot star wind spectra. Here we develop a parameterization that is as close as possible to the results of hydrodynamical calculations.

The most frequently used parameterization of the wind velocity law is the so-called beta velocity law,

$v(r)=v_{\infty, \beta}\left(1-\frac{R_{*}}{r}\right)^{\beta}$,

where $v_{\infty, \beta}$ is the wind terminal velocity, $\beta$ a free parameter describing the steepness of the velocity law, and $R_{*}$ the stellar radius. Thus, for parameterization of the wind velocity law using Eq. (1) we need two parameters, namely $\beta$ and the wind terminal velocity $v_{\infty, \beta}$. The popularity of the beta velocity law Eq. (1) stems from its simplicity and from the belief that the velocity profile described by Eq. (1) is close to the real one. Also simple analytical wind solutions have the form of Eq. (1). For example, in the case of accelerating force proportional to $\sim 1 / r^{2}$, the solution of the momentum equation (Milne 1926; Chandrasekhar 1934; Rublev 1965) leads to $v(r)=v_{\infty, \beta} \sqrt{1-R_{*} / r}$, implying $\beta=0.5$ in Eq. (1). Also the velocity law of a classical Castor et al. (1975) line-driven wind model can be approximated with $\beta=0.5$ (cf., Gayley 2000). Motivated by this, observational studies widely use Eq. (1) and usually consider $\beta$ as a free parameter, yielding a typical value of $\beta=0.7-1$ for $\mathrm{O}$ star winds (e.g., Puls et al. 1996). However, for some stars (e.g., early B supergiants), higher $\beta$ up to 3 may be determined (e.g. Crowther et al. 2006).

Several variants of the simple formula Eq. (1) can be found in the literature and generally aim to improve the fits of wind line profiles. For example, Hillier (1988) introduced the velocity law that also encompasses the wind part close to the hydrostatic photosphere. Subsequently, Hillier \& Miller (1999) introduced a second component in the beta law, which provides better fits to the blue edges of P Cygni line profiles. This was also supported by Gräfener \& Hamann (2005), who found that different values of $\beta$ describe the wind velocity law in different regions. The failure of the simple $\beta$-velocity law to describe the wind velocity law was also mentioned by Gayley et al. (1995).

Although the original beta velocity law was derived theoretically, there is no reason to believe that it provides the best approximation to the real situation. It is well known from hydrodynamical simulations (Feldmeier et al. 1997; Owocki \& Puls 1999) that the wind velocity field is variable with time, and consequently the beta law may at most describe mean flow. But even in stationary models, the wind velocity law may deviate from Eq. (1), at least because the wind is driven by different ions close to the star and at large radii (Vink et al. 2001; Krtička 2006).

It would be highly desirable to develop formulae that are able to describe the velocity law in a more accurate form than Eq. (1). It is not clear how to develop a more general formula based on Eq. (1). Luckily, the problem of functional fitting is fully settled in mathematics, where the concept of orthogonal functions was introduced. Consequently, we propose to use the set of polynomials that may form an orthogonal basis in a given functional space, e.g.,

$v(r)=\sum_{i=1}^{n} v_{i}\left(1-\frac{R_{*}}{r}\right)^{i}$,

from which the terminal velocity follows as $v_{\infty}=\sum_{i=1}^{n} v_{i}$. Equation (2a) can be equivalently rewritten in terms of Legendre 
Table 1. Best-fit parameters to the radial velocity from hydrodynamic models (Krtička \& Kubát 2010) for selected stars and individual velocity parameterizations.

\begin{tabular}{|c|c|c|c|c|c|c|c|c|c|c|}
\hline \multirow[t]{2}{*}{ Star } & \multirow[t]{2}{*}{ "HD number } & \multirow[b]{2}{*}{$\begin{array}{c}R_{*} \\
{\left[R_{\odot}\right]}\end{array}$} & \multirow[b]{2}{*}{$\begin{array}{c}M \\
{\left[M_{\odot}\right]}\end{array}$} & \multirow[b]{2}{*}{$\begin{array}{l}T_{\text {eff }} \\
{[\mathrm{K}]}\end{array}$} & \multicolumn{2}{|c|}{ 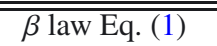 } & \multicolumn{4}{|c|}{ Eq. (2b) for $n=3$} \\
\hline & & & & & $\begin{array}{c}v_{\infty, \beta} \\
{\left[\mathrm{km} \mathrm{s}^{-1}\right]}\end{array}$ & $\beta$ & \multirow{2}{*}{\multicolumn{4}{|c|}{$\left[\mathrm{km} \mathrm{s}^{-1}\right]$}} \\
\hline & & & & O sta & & & & & & \\
\hline$\xi$ Per & HD 24912 & 14.0 & 36 & 35000 & 2130 & 0.70 & 1270 & 980 & -220 & 60 \\
\hline$\iota$ Ori & HD 37043 & 21.6 & 41 & 31400 & 2170 & 0.83 & 1190 & 1040 & -120 & 30 \\
\hline \multirow[t]{3}{*}{15 Mon } & HD 47839 & 9.9 & 32 & 37500 & 3240 & 0.90 & 1700 & 1590 & -90 & 20 \\
\hline & HD 54662 & 11.9 & 38 & 38600 & 2200 & 0.79 & 1230 & 1070 & -180 & -10 \\
\hline & HD 93204 & 11.9 & 41 & 40000 & 2210 & 0.79 & 1240 & 1070 & -170 & 0 \\
\hline$\zeta \mathrm{Oph}$ & HD 149757 & 8.9 & 21 & 32000 & 1940 & 0.85 & 1060 & 930 & -50 & 70 \\
\hline 68 Cyg & HD 203064 & 15.7 & 38 & 34500 & 1970 & 0.76 & 1120 & 920 & -150 & 50 \\
\hline 19 Cер & HD 209975 & 22.9 & 47 & 32000 & 2090 & 0.82 & 1160 & 1010 & -120 & 30 \\
\hline \multicolumn{11}{|c|}{ Central stars of planetary nebulae } \\
\hline NGC 2392 & & 1.5 & 0.41 & 40000 & 540 & 0.86 & 290 & 270 & -50 & -20 \\
\hline NGC 3242 & & 0.3 & 0.53 & 75000 & 2070 & 0.68 & 1260 & 920 & -290 & 60 \\
\hline IC 4637 & & 0.8 & 0.87 & 55000 & 1350 & 0.89 & 710 & 680 & -70 & -30 \\
\hline IC 4593 & & 2.2 & 1.11 & 40000 & 730 & 0.88 & 390 & 360 & -60 & -30 \\
\hline IC 418 & & 2.7 & 1.33 & 39000 & 800 & 0.87 & 420 & 380 & -70 & -20 \\
\hline Tc 1 & & 3.0 & 1.37 & 35000 & 910 & 0.95 & 460 & 440 & -30 & -10 \\
\hline NGC 6826 & & 2.2 & 1.40 & 44000 & 880 & 0.88 & 470 & 430 & -60 & -30 \\
\hline
\end{tabular}

Notes. The stellar parameters (radius $R_{*}$, mass $M$, and the effective temperature $T_{\text {eff }}$ ) are taken from Repolust et al. (2004), Markova et al. (2004), Pauldrach et al. (2004), and Martins et al. (2005).

polynomials. This guarantees that the derived coefficients decrease with increasing order $i$ and that the coefficients do not significantly change with increasing $n$ (e.g., Mikulášek 2007). In this case instead of Eq. (2a), the parameterizations is

$v(r)=\sum_{i=0}^{n} \tilde{v}_{i} \tilde{P}_{i}(x), \quad$ with $x=1-\frac{R_{*}}{r}$,

and the terminal velocity $v_{\infty}=\sum_{i=1}^{n} \tilde{v}_{i}$. To ensure the orthogonality in the interval $[0,1]$ we use shifted Legendre polynomials here. The first four are (e.g., Koornwinder et al. 2010)

$$
\begin{array}{ll}
\tilde{P}_{0}=1, & \tilde{P}_{1}=2 x-1, \\
\tilde{P}_{2}=6 x^{2}-6 x+1, \text { and } & \tilde{P}_{3}=20 x^{3}-30 x^{2}+12 x-1 .
\end{array}
$$

The relations between coefficients $\tilde{v}_{i}$ and $v_{i}$ follow from Eqs. (2) and (3)

$$
\begin{aligned}
\tilde{v}_{0} & =\frac{1}{2} v_{1}+\frac{1}{3} v_{2}+\frac{1}{4} v_{3}, & \tilde{v}_{1} & =\frac{1}{2} v_{1}+\frac{1}{2} v_{2}+\frac{9}{20} v_{3}, \\
\tilde{v}_{2} & =\frac{1}{6} v_{2}+\frac{1}{4} v_{3}, & \tilde{v}_{3} & =\frac{1}{20} v_{3} .
\end{aligned}
$$

Only three of these $\left(\tilde{v}_{i}\right)$ coefficients are independent.

To demonstrate the convenience of the formulas Eq. (2) we fit the radial velocity structure obtained from hydrodynamical NLTE wind models with CMF line force of Krtička \& Kubát (2010, see Fig. 1). The best-fit parameters of the beta velocity law and polynomial fit Eq. (2b) with $n=3$ for individual stars are given in Table 1. To test the global accuracy of individual orders of the velocity approximation Eq. (2), we plot the relative difference between the velocity from the numerical models $v^{\text {mod }}$ and its approximations $v^{\text {app }}$ (Eq. (2b) for $n=1,2,3$ and Eq. (1)) averaged over individual stars in Table $1,\left\langle\left|\left(v^{\text {mod }}-v^{\text {app }}\right) / v^{\text {app }}\right|\right\rangle$, as a function of radius in Fig. 2.

Already the first term in Eq. (2a), i.e. the approximation $v(r)=v_{1}\left(1-R_{*} / r\right)$ (in fact Eq. (1) for $\beta=1$ ), provides reasonable approximation to the wind velocity law suitable for simplified analysis (see Fig. 2). Including the second term in Eq. (2a)

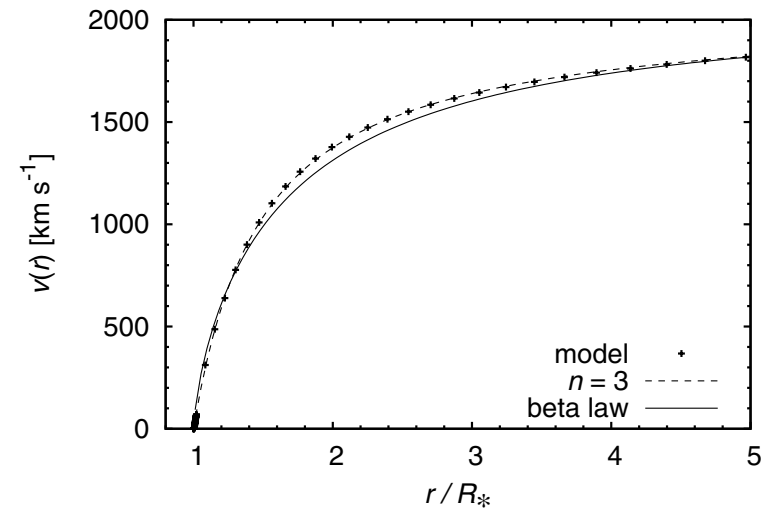

Fig. 1. Comparison of the fit to the calculated hydrodynamical model radial velocity of HD 24912 (crosses) by the beta velocity law (solid line), and by the polynomial fit Eq. (2b) for $n=3$.

we obtain better approximation than the usual beta velocity law both for the velocity and its derivative using the same number of free parameters $\left(\beta\right.$ and $v_{\infty, \beta}$ for the beta velocity law, $v_{1}$ and $v_{2}$ for Eq. (2a)). The agreement in the outer parts of the wind $\left(r / R_{*} \gtrsim 1.1\right)$ can be improved further by adding the third term in Eq. (2a) (see Fig. 2).

It has not escaped our attention that even relatively high order polynomials do not provide reasonable fits in the region close to the star, for $r / R_{*} \lesssim 1.1$. We do not aim to fit the velocity in this region, because here the wind density approaches the hydrostatic density stratification. Consequently, it would be better to derive the velocity from the continuity equation using density from the hydrostatic equilibrium equation instead of a polynomial.

Many studies fit the observed line profiles of particular stars using sophisticated radiative transfer calculations (e.g., Puls et al. 1996; Hillier \& Miller 1999) by assuming the velocity law of type Eq. (1) and trying to find the most suitable value of the parameter $\beta$ in combination with the value of the terminal velocity $v_{\infty}$. Although our formulae Eq. (2) were derived to 


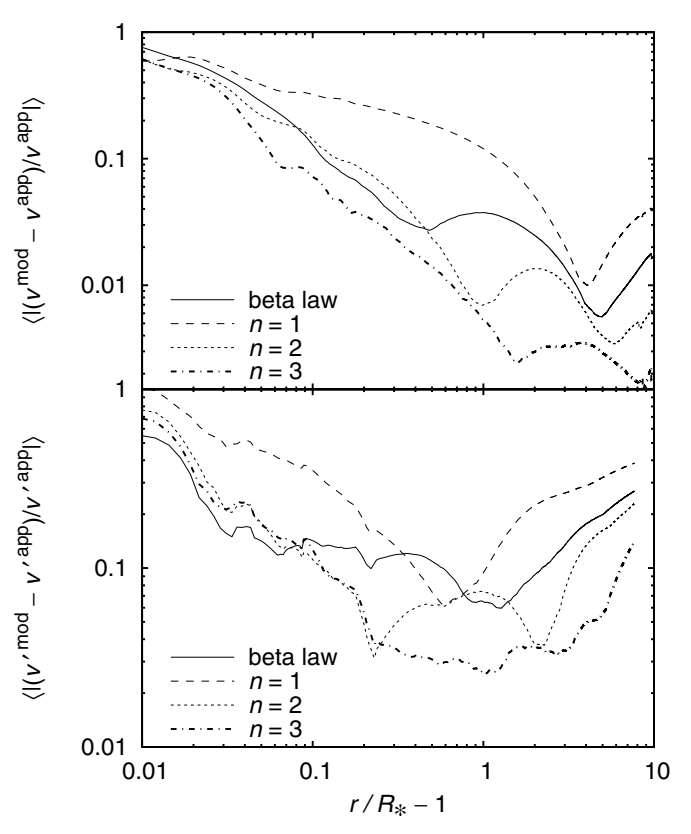

Fig. 2. The mean relative difference (see text) between the velocity law (upper panel) and its derivative $v^{\prime}=\mathrm{d} v / \mathrm{d} r$ (bottom panel) taken from hydrodynamical models and its approximations averaged over stars from Table 1 as a function of radius.

fit the results of numerical hydrodynamical calculations, it might be interesting to use them to also fit the beta velocity profiles. The test showed that the polynomial approximation Eq. (2) with $n=2$ provides very good fits to beta laws (Eq. (1)) both with small $\beta<1$ and large $\beta>1$ and to the combined law of Hillier \& Miller (1999). Only for large $\beta \gtrsim 2.3$ is it better to increase the polynomial degree $(n=3)$ to assure the positivity of the fit close to the star. The modified beta law $v(r)=v_{\infty, \beta}\left(1-b \frac{R_{*}}{r}\right)^{\beta}$ with additional parameter $b<1$ (Puls \& Hummer 1988) is frequently used to improve the fit close to the star. It can be satisfactorily reproduced by either introducing a fixed low value of $v_{0}$ in Eq. (2a) or by a substitution $x=1-b R_{*} / r$ in Eq. (2b). Moreover, our experiments with fitting the trial P Cygni line profiles have shown that the fits using Eq. (2) and the beta velocity law require a comparable number of line profile evaluations. A reasonable fit strategy could be to derive $v_{\infty}$ from observations and then keep the sum $\sum_{i=1}^{n} v_{i}=v_{\infty}$ fixed.

We conclude that the beta velocity law provides reasonable approximation to the hydrodynamical wind velocity law and its derivative. The approximation can be improved with polynomial fits Eq. (2a) (or, equivalently Eq. (2b)) using the same number of free parameters as in the commonly used beta velocity law.

Acknowledgements. We thank Prof. Achim Feldmeier and Dr. Joachim Puls for discussions of this subject. This work was supported by grant GA ČR 205/08/0003. The Astronomical Institute Ondřejov is supported by the project AV0Z10030501.

\section{References}

Castor, J. I., Abbott, D. C., \& Klein, R. I. 1975, ApJ, 195, 157 (CAK) Chandrasekhar, S. 1934, MNRAS, 94, 522

Crowther, P. A., Lennon, D. J., \& Walborn, N. R. 2006, A\&A, 446, 279

Feldmeier, A., Puls, J., \& Pauldrach, A. W. A. 1997, A\&A, 322, 878

Gayley, K. G. 2000, ApJ, 529, 1019

Gayley, K. G., Owocki, S. P., \& Cranmer, S. R. 1995, ApJ, 442, 296

Gräfener, G., \& Hamann, W.-R. 2005, A\&A, 432, 633

Hillier, D. J. 1988, ApJ, 327, 822

Hillier, D. J., \& Miller, D. L. 1999, ApJ, 519, 354

Koornwinder, T. H., Wong, R. S. C., Koekoek, R., \& Swarttouw, R. F. 2010, in NIST Handbook of Mathematical Functions, ed. F. W. J. Olver, D. M. Lozier, R. F. Boisvertet et al. (Cambridge: Cambridge University Press) Krtička, J. 2006, MNRAS, 367, 1282

Krtička, J., \& Kubát, J. 2010, A\&A, 519, A50

Markova, N., Puls, J., Repolust, T., \& Markov, H. 2004, A\&A, 413, 693

Martins, F., Schaerer, D., Hillier, D. J., et al. 2005, A\&A, 441, 735

Mikulášek, Z. 2007, Odessa Astronomical Publications, 20, 138

Milne, E. A. 1926, MNRAS, 86, 459

Owocki, S. P., \& Puls, J. 1999, ApJ, 510, 355

Pauldrach, A. W. A., Hoffmann, T. L., \& Méndez, R. H. 2004, A\&A, 419, 111

Puls, J., \& Hummer, D. G. 1988, A\&A, 191, 87

Puls, J., Kudritzki, R.-P., Herrero, A., et al. 1996, A\&A, 305, 171

Repolust, T., Puls, J., \& Herrero, A. 2004, A\&A, 415, 349

Rublev, S. V. 1965, AZh, 42, 718 (SvA, 9, 555)

Vink, J. S., de Koter, A., \& Lamers, H. J. G. L. M. 2001, A\&A, 369, 574 